Check for updates

Cite this: RSC Adv., 2018, 8, 26849

\title{
Synergistic effect of temperature and background counterions on ion-exchange equilibria
}

\author{
Masami Shibukawa, (D) *a Masaru Yanagisawa, ${ }^{a}$ Ryota Morinaga, ${ }^{a}$ Tomomi Shimasaki, ${ }^{a}$ \\ Shingo Saito, (D) ${ }^{\text {a }}$ Shao-ting Wang ${ }^{\mathrm{b}}$ and Yu-qi Feng (DD ${ }^{\mathrm{b}}$
}

The effects of temperature and background counterions on ion-exchange selectivity for alkali metal ions and tetraalkylammonium ions on strongly acidic cation-exchange resins have been investigated using superheated water ion-exchange chromatography (SW-IEC). We have found out that alkali metal ions show reversal in the order of the distribution coefficient $\left(K_{\mathrm{D}}\right)$, from $\mathrm{Li}^{+}<\mathrm{Na}^{+}<\mathrm{K}^{+}<\mathrm{Rb}^{+}$in water at ordinary temperature to $\mathrm{Rb}^{+}<\mathrm{K}^{+}$ $<\mathrm{Na}^{+}<\mathrm{Li}^{+}$in superheated water, when a relatively large cation such as cesium ion is used as the background counterion. The effect of counterion on the ion-exchange selectivity is enhanced with the ion-exchange resins of higher ion-exchange capacity and cross-linking degree. Tetraalkylammonium ions chosen as model ions for poorly hydrated ions also exhibit reversal in the order of $K_{\mathrm{D}}$ at around $430 \mathrm{~K}$ in superheated water. However, the effect of the nature of alkali metal counterions on the change in $K_{D}$ values of tetraalkylammonium ions is rather small compared with the effect on the $K_{\mathrm{D}}$ of alkali metal ions. These results are attributed to the change in local hydration structures of the ions in the ion-exchange resin due to dehydration of alkali metal ions enhanced by interionic contacts of the analyte ion with the coexisting counterion and lower hydration energy of the ions at elevated temperatures. Although it has been considered that temperature is not effective at changing the ion-exchange separation selectivity, significant selectivity changes can be achieved by SW-IEC.

Received 18th April 2018

Accepted 20th July 2018

DOI: $10.1039 / \mathrm{c} 8 \mathrm{ra03309a}$

rsc.li/rsc-advances where $\mathrm{s}$ and $\mathrm{r}$ refer to the solution and exchanger phases, respectively. The selectivity of an ion-exchange separation may be expressed as the separation factor for a pair of analyte ions $\mathbf{A}$ and $\mathbf{B}, \alpha_{\mathbf{A} / \mathbf{B}}$, which is defined by the equation:

$$
\alpha_{\mathbf{A} / \mathbf{B}}=K_{\mathrm{D}}(\mathbf{A}) / K_{\mathrm{D}}(\mathbf{B})
$$

where $K_{\mathrm{D}}(\mathbf{A})$ and $K_{\mathrm{D}}(\mathbf{B})$ are the distribution coefficients of ions $\mathbf{A}$ and $\mathbf{B}$ between the solution and exchanger phases, respectively. If it can be assumed that the distribution of the analyte ions takes place solely by displacement of the counterion on the fixed ions through the ion-exchange reaction represented by eqn (1), the distribution coefficient of ion $\mathbf{A}$ is expressed as

$$
K_{\mathrm{D}}(\mathbf{A})=\frac{[\mathbf{A}]_{(\mathrm{r})}}{[\mathbf{A}]_{(\mathrm{s})}}=K_{\mathrm{D}}(\mathbf{E})^{\frac{z_{\mathbf{A}} \mid}{\mid z_{\mathrm{E}}}} K_{\mathbf{A} / \mathbf{E} \mid}^{\mathrm{c}} \frac{1}{z_{\mathbf{E}} \mid}
$$
in turn releases different counterions of like sign. Therefore the stoichiometric ion-exchange reaction between an analyte ion $\mathbf{A}^{z_{A}}$ and the counterion of the fixed charged groups of the exchanger $\mathbf{E}^{Z_{\mathbb{E}}}$ may be written as ${ }^{1}$

$$
z_{\mathbf{E}} \mathbf{A}^{z_{A}}(\mathrm{~s})+z_{\mathbf{A}} \mathbf{E}^{z_{\mathrm{E}}}(\mathrm{r}) \rightleftarrows z_{\mathbf{E}} \mathbf{A}^{z_{A}}(\mathrm{r})+z_{\mathbf{A}} \mathbf{E}^{z_{\mathbf{E}}}(\mathrm{s})
$$

${ }^{a}$ Graduate School of Science and Engineering, Saitama University, 255 Shimo-Okubo, Sakura-ku, Saitama 338-8570, Japan. E-mail: sibukawa@apc.saitama-u.ac.jp

${ }^{b}$ Key Laboratory of Analytical Chemistry for Biology and Medicine (Ministry of Education), Department of Chemistry, Wuhan University, Wuhan 430072, P. R. China where $[\mathbf{A}]_{(\mathrm{s})}$ and $[\mathbf{A}]_{(\mathrm{r})}$ are the concentrations of the analyte ion $\mathbf{A}^{z_{A}}$ in the solution and exchanger phases, $K_{\mathrm{D}}(\mathbf{E})$ is the distribution coefficient of the counterion $\mathbf{E}^{z_{\mathbf{E}}}$, and $K_{\mathbf{A} / \mathbf{E}}^{\mathrm{c}}$ is the molar selectivity coefficient for the ion-exchange equilibrium of ions $\mathbf{A}^{z_{A}}$ and $\mathbf{E}^{z_{\mathrm{E}}}$, respectively. Substituting the distribution coefficients for ions $\mathbf{A}^{z_{\boldsymbol{A}}}$ and $\mathbf{B}^{z_{\mathbf{B}}}$ with equal charge $\left(z_{\mathbf{A}}=z_{\mathbf{B}}\right)$ given by eqn (3) into eqn (2), we have

$$
\alpha_{\mathbf{A} / \mathbf{B}}=\left(\frac{K_{\mathbf{A} / \mathbf{E}}^{\mathrm{c}}}{K_{\mathbf{B} / \mathbf{E}}^{\mathrm{c}}}\right)^{\frac{1}{\left|z_{\mathbf{E}}\right|}}=K_{\mathbf{A} / \mathbf{B}}^{\mathrm{c}}
$$


This equation indicates that the separation factor or the selectivity coefficient of a pair of analyte ions of equal charge should be constant independent of the nature of the background counterion. However, it has been known that the selectivity coefficient is characteristic of the nature and concentration of the counterion even when the other equilibria than ionexchange such as complexation of the analyte ion with coexisting species in the system and specific adsorption onto the surface of the exchanger matrix are negligible. ${ }^{\mathbf{1 , 4}-\mathbf{8}}$ This reveals that the selectivity coefficient cannot be regarded as a thermodynamic equilibrium constant but depends on the nature of coexisting counterions particularly in the exchanger phase.

Most of the studies that have so far been devoted to the prediction of the selectivity coefficient are based on empirical or semi-empirical estimation of the activity coefficients of the chemical species in the solution and the exchanger. Several physicochemical models have also been set up on the basis of electrical double layer theories to estimate the activity coefficient of the analyte ion in the exchanger phase. ${ }^{4-13}$ However, no rational explanation has yet been given with regard to the dependence of the selectivity coefficient on the type of the counterion.

It must be noted that the interaction of ions with water molecules or hydration is always involved in ion-exchange processes whatever the main mechanisms contributing to the separation are. In particular, it is essential to clarify the manner in which the hydration of ions is affected by the presence of the coexisting counterion in the exchanger phase in order to understand the change in the separation factor with the nature of background counterion.

We recently developed superheated water ion-exchange chromatography (SW-IEC) that uses liquid water under pressure at temperatures above $100{ }^{\circ} \mathrm{C}$ and clarified that the change in hydration structure of ions with temperature causes a drastic change in the ion-exchange separation selectivity. ${ }^{\mathbf{1 4 - 1 6}}$ For example, the selectivity coefficient for a pair of alkali metal ions or that of halide ions approaches unity as temperature increases. Therefore the distribution coefficient of an analyte ion decreases with an increase in temperature when the background eluent counterion in the mobile phase has stronger affinity than that of the analyte ion for the ion-exchanger, whereas it increases when eluted by a weaker counterion. ${ }^{\mathbf{1 4 - 1 8}}$ Presuming that the dependence of ion-exchange selectivity observed for simple inorganic ions on temperature can be attributed to the change in hydration of the ions in the solution phase and/or in the ion-exchange resin with temperature, we investigated the effect of temperature on the first-shell coordination structures of $\mathrm{Rb}^{+}, \mathrm{Sr}^{2+}, \mathrm{Br}^{-}$and $\mathrm{I}^{-}$in aqueous solution and those for $\mathrm{Rb}^{+}$and $\mathrm{Sr}^{2+}$ in a sulfonated styrenedivinylbenzene copolymer cation-exchange resin in the temperature range from ambient temperature to $450 \mathrm{~K}$ by X-ray absorption fine structure (XAFS) spectroscopy. ${ }^{19}$ The spectral changes observed for these ions indicate that the number of coordinated first shell water molecules decreases with an increase in temperature. It has thus been concluded that the effect of temperature on separation selectivity in ion-exchange processes can be interpreted by the change in hydration of ions due to disruption of ion-dipole bonding by thermal motion of water molecules, which makes the charge and the size of an ion decisive factors determining the separation selectivity in ion-exchange processes at elevated temperatures.

On the other hand, the experimental results obtained by SWIEC reveal that not only the ion-exchange selectivity itself but also its temperature dependence varies depending on the nature of the co-existing background counterion. ${ }^{\mathbf{1 4 , 1 5}}$ This suggests that the counterion may affect the hydration structure of the analyte ion in the internal region of the ion-exchange resin. However, the origin of the effect of the co-existing counterion on the ion-exchange selectivity has not been clarified yet. In the present work, the effect of elevated temperature on the distribution coefficients of alkali metal ions and tetraalkylammonium ions on strongly acidic sulfonated ion exchangers has been examined over a wide temperature range using SWIEC with aqueous solutions of alkali metal and tetraalkylammonium salts. We will show that the effect of the co-existing counterion on the ion-exchange selectivity is attributable to the change in the ion hydration structure caused by the counterion in the resin. A drastic change in separation selectivity of ionic solutes will also be demonstrated using a synergistic effect of temperature and the counterion on the hydration structure of analyte ions.

\section{Experimental}

\section{Chemicals}

All chemicals used in this study were obtained from commercial sources and were of analytical reagent-grade unless otherwise stated. Deuterated water $\left(\mathrm{D}_{2} \mathrm{O}\right)$ for NMR use was purchased from Wako Pure Chemicals (Tokyo, Japan) and Blue Dextran 2000 was obtained from Pharmacia Fine Chemicals (Uppsala, Sweden). Water was purified subsequently with an ion-exchange cartridge PF-III H10 (Organo, Tokyo, Japan) and an Arium 611 DI (Sartorius, Tokyo, Japan). The cation-exchangers used were sulfonated polystyrene-divinylbenzene copolymer resins, DIAION MCI GEL CK10S (degree of crosslinking $=10 \%$, particle diameter $=11 \mu \mathrm{m}$ ) and CK02A (degree of crosslinking $=2 \%$, particle diameter $=20 \mu \mathrm{m})$, purchased from Mitsubishi Chemical (Tokyo, Japan), and a tetrafluoroethylene-perfluoro[2(fluorosulfonylethoxy)-propylvinylether] copolymer resin, Nafion (35-60 mesh), from Sigma-Aldrich Japan (Tokyo, Japan). The $\mathrm{Na}^{+}$form of MCI GEL CK10S or CK02A was slurry packed into a stainless steel column with water. The $\mathrm{H}^{+}$form of Nafion was converted to the $\mathrm{Na}^{+}$form by decantation with $50 \mathrm{mM}$ $\mathrm{Na}_{2} \mathrm{SO}_{4}$ and then packed into a stainless steel column with the same solution.

\section{Determination of cation-exchange capacities of the cation- exchange resin columns}

The ion-exchange capacity of the MCI GEL CK10S column was determined by titrating hydrogen ion eluted from the $\mathrm{H}^{+}$form resin column by passing a sufficient amount of $\mathrm{Na}_{2} \mathrm{SO}_{4}$ solution with standard $\mathrm{NaOH}$ solution. On the other hand, the ionexchange capacities of the MCI GEL CK02A and the Nafion 
columns were measured in the following manner because the value of the ion-exchange capacity is rather small. An aqueous solution containing $25 \mathrm{mM} \mathrm{Li} \mathrm{SO}_{4}$ was passed through the column at the flow rate of $0.5 \mathrm{~mL} \min ^{-1}$ for $2 \mathrm{~h}$. After the column was washed with pure water at the flow rate of 0.5 $\mathrm{mL} \min ^{-1}$ for $1 \mathrm{~h}, 10 \mathrm{mM} \mathrm{K} \mathrm{SO}_{4}$ solution was passed into the column at the flow rate of $0.5 \mathrm{~mL} \mathrm{~min}^{-1}$ for $3 \mathrm{~h}$ and the effluent from the column was collected into a $100 \mathrm{~mL}$ volumetric flask. The concentration of lithium ion in the solution was determined by means of an inductively coupled plasma atomic emission spectrometer, OPTIMA 5300DV (Perkin Elmer, Yokohama, Japan). The capacity of every column used for the study was determined just after a series of experiments conducted with superheated water.

\section{SW-IEC conditions}

Chromatographic measurements were performed on an HPLC system consisting of a GL Science (Tokyo, Japan) Model DG660 degasser, a Hitachi (Tokyo, Japan) Model L-6000 pump, a Rheodyne (Cotati, CA, USA) Model 7215 loading injector fitted with a 1 or $20 \mu \mathrm{L}$ sample loop, and a Showa Denko (Tokyo, Japan) Model Shodex RI-101 refractometric detector. A preheating coil (Hastelloy, $0.5 \mathrm{~m} \times 0.5 \mathrm{~mm}$ I.D.) and a backpressure coil (stainless steel, $1.5 \mathrm{~m} \times 0.1 \mathrm{~mm}$ I.D.) were placed between the injector and the column inlet and between the column outlet and the detector, respectively. The preheating coil and the column were thermostated using a Sandra Selerity Technologies (Salt Lake City, UT, USA) Model Polaratherm Series 9000 column temperature controller for SWC. The temperature of the oven was monitored with a mercury thermometer inserted into the oven.

Aqueous solutions of alkali metal sulfates or tetramethyammonium bromide were used as eluents. All the eluents were filtered through a $0.45 \mu \mathrm{m}$ membrane filter and degassed with an aspirator in an ultrasonic bath, US CLEANER US-2R (AS ONE, Tokyo, Japan) before use.

The extra column volume was determined by measuring the elution volume of a sample solute through the system from which the column had been removed. The volumetric flow rates $F$ were measured using a volumetric flask and the exact values of the flow rates $F^{\prime}$ in the column were calculated according to the following equation:

$$
F^{\prime}=\frac{\rho_{\mathrm{r}}}{\rho_{\mathrm{T}}} F
$$

where $\rho_{\mathrm{r}}$ and $\rho_{\mathrm{T}}$ are the density of liquid water at room temperature and at the column temperature, respectively. ${ }^{30}$ The retention volume $\boldsymbol{V}_{\mathrm{R}}$ of an analyte ion was obtained from the values of $F$ and $F^{\prime}$ and the retention time $t_{\mathrm{R}}$ as follows:

$$
\boldsymbol{V}_{\mathrm{R}}=\left(\boldsymbol{t}_{\mathrm{R}}-\frac{\boldsymbol{V}_{\mathrm{ex}}}{F}\right) F^{\prime}
$$

where $\boldsymbol{V}_{\text {ex }}$ is the extra column volume.

Test solutions were prepared by dissolving analyte compounds in the eluent to be used. The retention volumes for each analyte ion was measured in the temperature range of 273$473 \mathrm{~K}$ for the MCI GEL CK10S column, 298-403 K for the MCI
GEL CK02A column and 308-423 $\mathrm{K}$ for the Nafion column, respectively. The detection signal was fed into a CDS plus data analysis system (LA Soft, Tokyo, Japan).

\section{Measurement of temperature-dependent swelling properties of a cation-exchange resin}

The $\mathrm{Na}^{+}$form of MCI GEL CK10S was slurry packed into a stainless steel column $(150 \mathrm{~mm} \times 4.6 \mathrm{~mm}$ i.d. $)$ with water. $\mathrm{The}^{+}, \mathrm{Na}^{+}, \mathrm{K}^{+}, \mathrm{Rb}^{+}$and $\mathrm{Cs}^{+}$forms of the cation-exchange resin column were prepared by passing $120 \mathrm{~mL}$ of aqueous solutions containing the respective metal sulfates $(25 \mathrm{mM})$ through the column at $60{ }^{\circ} \mathrm{C}$ and then washing the column with water. The total water volume and the interstitial volume or interparticulate volume in the column were determined by measuring the retention volumes of $\mathrm{D}_{2} \mathrm{O}$ and Blue Dextran 2000 , respectively.

\section{Results and discussion}

\section{Effects of temperature and counterion on the distribution coefficients of alkali metal ions and tetraalkylammonium ions}

Assuming that the mobile phase is the space occupied by water in the column and the stationary phase is the other part of the column, i.e., the resin matrix, we calculated the $K_{\mathrm{D}}$ value of an analyte ion by the following equation:

$$
K_{\mathrm{D}}=\frac{\boldsymbol{V}_{\mathrm{R}}-\boldsymbol{V}_{0}}{\boldsymbol{V}_{\mathrm{s}}}=\frac{\boldsymbol{V}_{\mathrm{R}}-\boldsymbol{V}_{0}}{\boldsymbol{V}_{\mathrm{t}}-\boldsymbol{V}_{0}}
$$

where $\boldsymbol{V}_{0}$ is the retention volume of $\mathrm{D}_{2} \mathrm{O}$ and $\boldsymbol{V}_{\mathrm{s}}$ is the volume of the ion-exchange resin obtained by subtracting the $V_{0}$ value from the total column volume $\boldsymbol{V}_{\mathrm{t}}$. Fig. 1(a) and (b) show the van't Hoff plots for alkali metal ions on a column packed with a sulfonated polystyrene-divinylbenzene copolymer resin, MCI

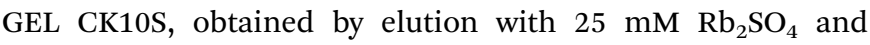
$\mathrm{Cs}_{2} \mathrm{SO}_{4}$ aqueous solutions, respectively. The $K_{\mathrm{D}}(\mathrm{E})$ for each system was estimated by

$$
K_{\mathrm{D}}(\mathbf{E})=\frac{\boldsymbol{Q}}{\left|z_{\mathbf{E}}\right| \boldsymbol{V}_{\mathrm{s}}[\mathbf{E}]_{(\mathrm{s})}}
$$

where $\boldsymbol{Q}$ is the ion-exchange capacity of the column used. The $K_{\mathrm{D}}(\mathbf{E})$ value is shown as a solid line in Fig. 1 . It should be noted that all the plots are nonlinear and the reversal in the retention order of alkali metal ions was observed at a higher temperature. When cesium was used as the eluent counterion, in particular, the order of $K_{\mathrm{D}}$ values for alkali metal ions was completely reversed by increasing temperature from $273 \mathrm{~K}_{\left(\mathrm{Li}^{+}<\mathrm{Na}^{+}<\mathrm{K}^{+}<\right.}$ $\left.\mathrm{Rb}^{+}\right)$to $430 \mathrm{~K}\left(\mathrm{Rb}^{+}<\mathrm{K}^{+}<\mathrm{Na}^{+}<\mathrm{Li}^{+}\right)$and lithium showed much larger $K_{\mathrm{D}}$ value than the other alkali metal ions at $370 \mathrm{~K}$ or above. To our best knowledge, it is the first time ever that this very interesting phenomenon has been found in pure aqueous system.

We reported in a previous paper that the $K_{\mathrm{D}}$ values of alkali metal ions on the MCI GEL CK10S column appear to converge on the $K_{\mathrm{D}}(\mathbf{E})$ value or the $K_{\mathbf{A} / \mathbf{E}}^{\mathrm{c}}$ approaches unity with an increase in temperature when $\mathrm{Li}^{+}, \mathrm{Na}^{+}$or $\mathrm{K}^{+}$is used as the eluent ion. ${ }^{\mathbf{1 4}}$ The results shown in Fig. 1, however, indicate that the selectivity 

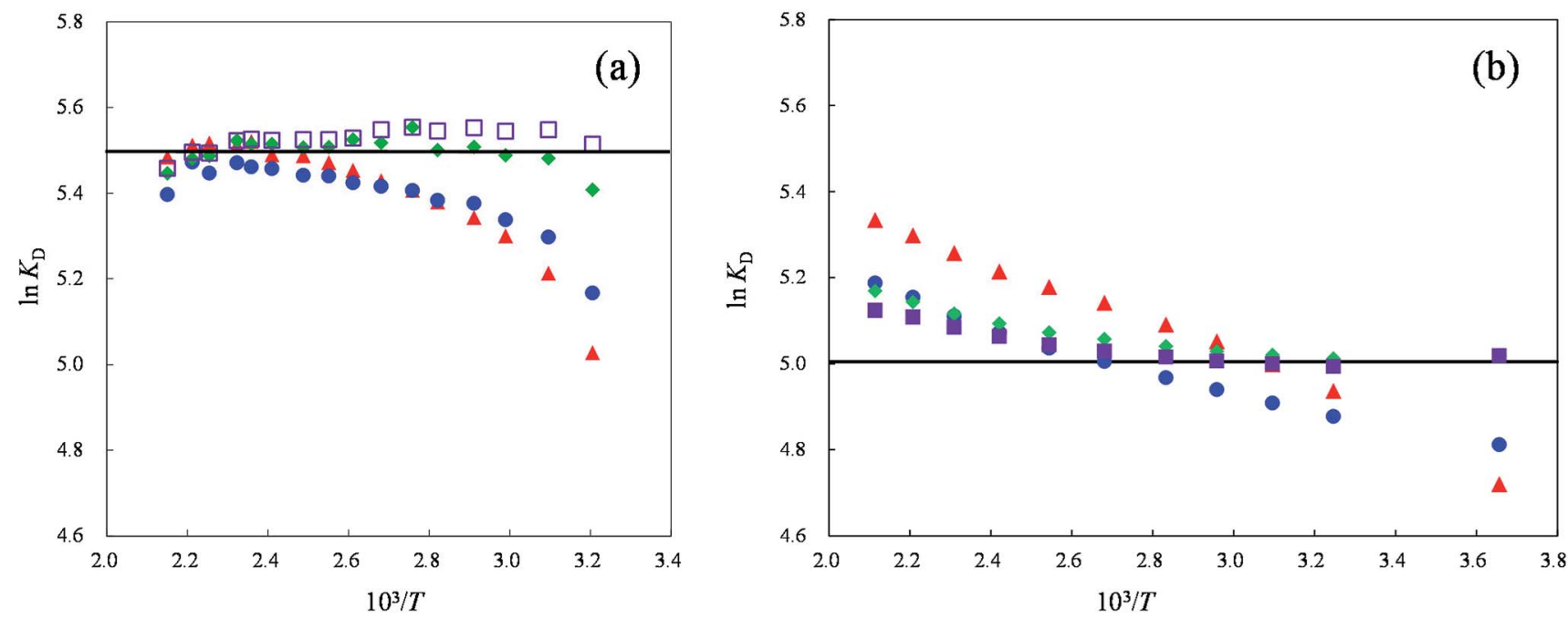

Fig. 1 Plots of $\ln K_{\mathrm{D}}$ for alkali metal ions as a function of reciprocal of temperature on a $\mathrm{MCl} G E L C K 10 S$ column. Eluent: (a) $25 \mathrm{mM} \mathrm{Rb}_{2} \mathrm{SO}_{4}$, (b) $25 \mathrm{mM} \mathrm{Cs}_{2} \mathrm{SO}_{4}$. Symbols: $(\Delta) \mathrm{Li}^{+},(\bullet) \mathrm{Na}^{+},(\diamond) \mathrm{K}^{+},(\square) \mathrm{Rb}^{+},(\subset) \mathrm{Cs}^{+}$. The solid line denotes the In $K_{\mathrm{D}}(\mathrm{E})$ value for each column.

coefficient does not approach unity with an increase in temperature but is reversed at a temperature specific to the background counterion. The reversal of the order of $K_{\mathrm{D}}$ for $\mathrm{Li}^{+}$ and $\mathrm{Na}^{+}$occurs at around $290 \mathrm{~K}$ in the $\mathrm{Cs}_{2} \mathrm{SO}_{4}$ eluent system, while it does at $360 \mathrm{~K}$ in the $\mathrm{Rb}_{2} \mathrm{SO}_{4}$ system.

We clarified by XAFS measurements that hydration of alkali metal ions and halide ions in the ion-exchange resin as well as aqueous solutions becomes weaker as the temperature rises due to disruption of ion-dipole bonding by thermal motion of water molecules. ${ }^{19}$ We thus concluded that the ion-exchange selectivity for simple inorganic ions decreases with an increase in temperature by the reduction of the difference in hydration structure between different ions. However, the $\ln K_{\mathrm{D}}$ vs. 1/T plots shown in Fig. 1 suggest that the coexisting counterion may also affect the hydration structures of analyte ions resulting in the drastic change in ion-exchange selectivity due to the synergistic effect of temperature and the background counterion.

We have shown that smaller tetraalkylammonium ion with a shorter alkyl chain exhibits lower $K_{\mathrm{D}}$ value at lower temperatures, while at higher temperatures the larger ion is eluted faster than the smaller one, indicating that the electrostatic interaction is a predominant mechanism and then ionic size is a determinant in retention in SW-IEC. ${ }^{14}$ Taking into account the fact that the order of hydrated ionic radii for alkali metal ions at ordinary temperature is $\mathrm{Rb}^{+}<\mathrm{K}^{+}<\mathrm{Na}^{+}<\mathrm{Li}^{+}$, whereas that of crystal ionic radii is $\mathrm{Li}^{+}<\mathrm{Na}^{+}<\mathrm{K}^{+}<\mathrm{Rb}^{+},{ }^{20,21}$ it is presumed that a similar phenomenon to that of tetraalkylammonium ion may also be observed even for relatively strongly hydrated alkali metal ions by the synergistic effect of temperature and counterion when cesium is used as the background eluent ion.

Fig. 2 shows the $\ln K_{\mathrm{D}} v s .1 / T$ plots for tetraalkylammonium ions when eluted with $25 \mathrm{mM} \mathrm{Cs}_{2} \mathrm{SO}_{4}$ solution. Although the temperature at which the reversal of retention order occurs appears to be a little lower in $\mathrm{Cs}_{2} \mathrm{SO}_{4}$ eluent system than in $\mathrm{Na}_{2} \mathrm{SO}_{4}$ system, ${ }^{14}$ the effect of the nature of alkali metal counterions on the change in retention of tetraalkylammonium ions is not very large. This is probably because tetraalkylammonium ions are poorly hydrated even at lower temperatures. The SW-IEC chromatograms obtained by elution with $25 \mathrm{mM} \mathrm{Cs}_{2} \mathrm{SO}_{4}$ aqueous solution are shown in Fig. 3 and 4 for mixtures of alkali metal ions and tetraalkylammonium ions, respectively. It will be noted that lithium is selectively separated from the other alkali metal ions and the retention order of tetraalkylammonium ions is completely reversed in superheated water.

Fig. 5(a) and (b) show the $\ln K_{\mathrm{D}} v s .1 / T$ plots for alkali metal ions obtained by elution with $25 \mathrm{mM} \mathrm{Cs}_{2} \mathrm{SO}_{4}$ aqueous solution

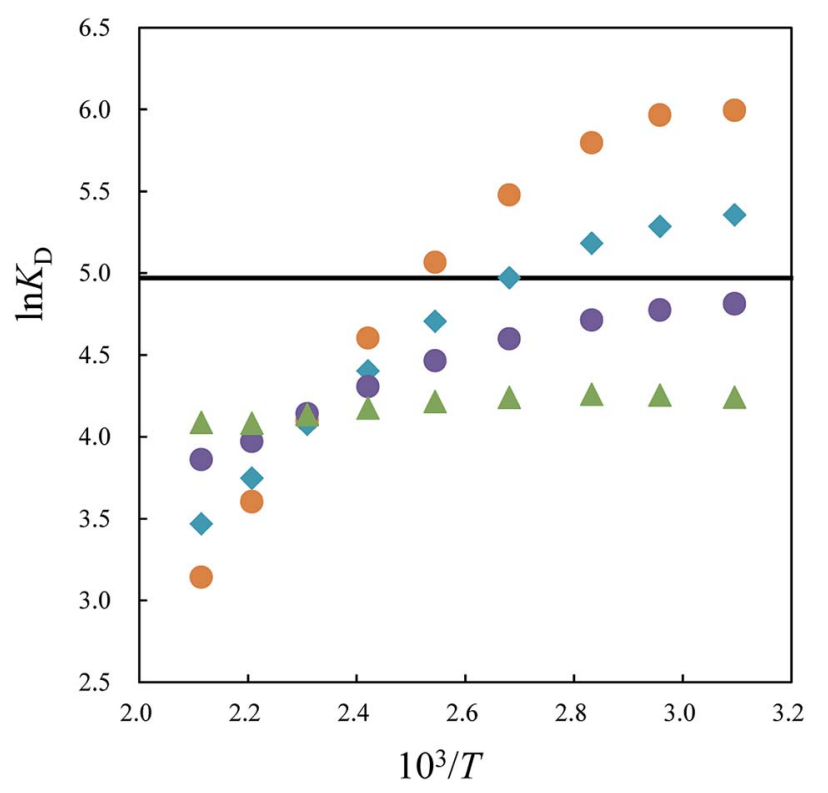

Fig. 2 Plots of $\ln K_{\mathrm{D}}$ for tetraalkylammonium ions as a function of reciprocal of temperature on a $\mathrm{MCI}$ GEL CK10S column. Eluent: $25 \mathrm{mM}$ $\mathrm{CS}_{2} \mathrm{SO}_{4}$. ( $\Delta$ ) tetramethylammonium ion, (๑) tetraethylammonium ion, $(\diamond)$ tetra- $n$-propylammonium ion, (๑) tetra- $n$-butylammonium ion. The solid line denotes the $\ln K_{\mathrm{D}}(\mathrm{E})$ value. 


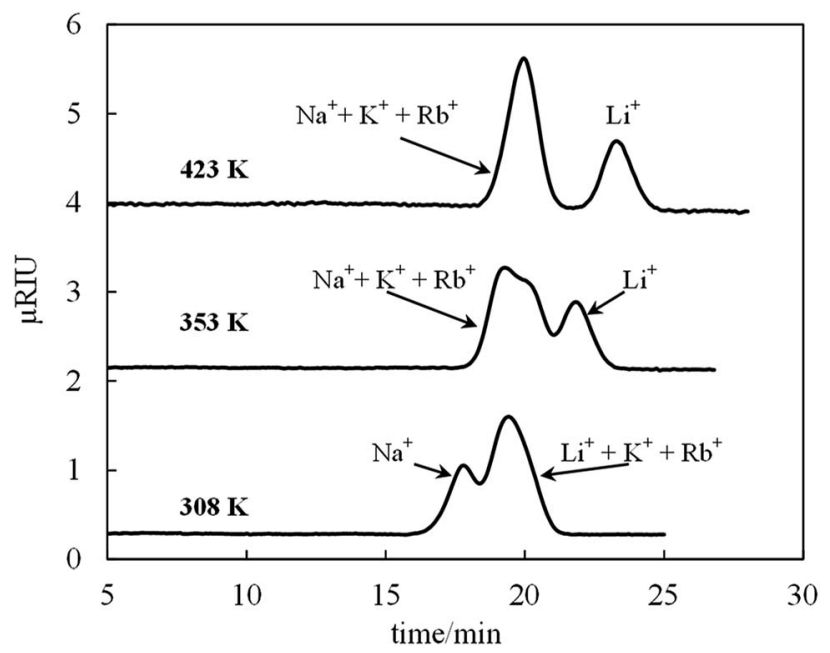

Fig. 3 Chromatograms of alkali metal ions on a MCl GEL CK10S column $(4.6 \mathrm{~mm}$ I.D. $\times 50 \mathrm{~mm})$ obtained at $308 \mathrm{~K}, 353 \mathrm{~K}$ and $423 \mathrm{~K}$. Eluent: $25 \mathrm{mM} \mathrm{Cs}_{2} \mathrm{SO}_{4}$. Detection: differential reflactometer. Flow

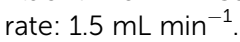

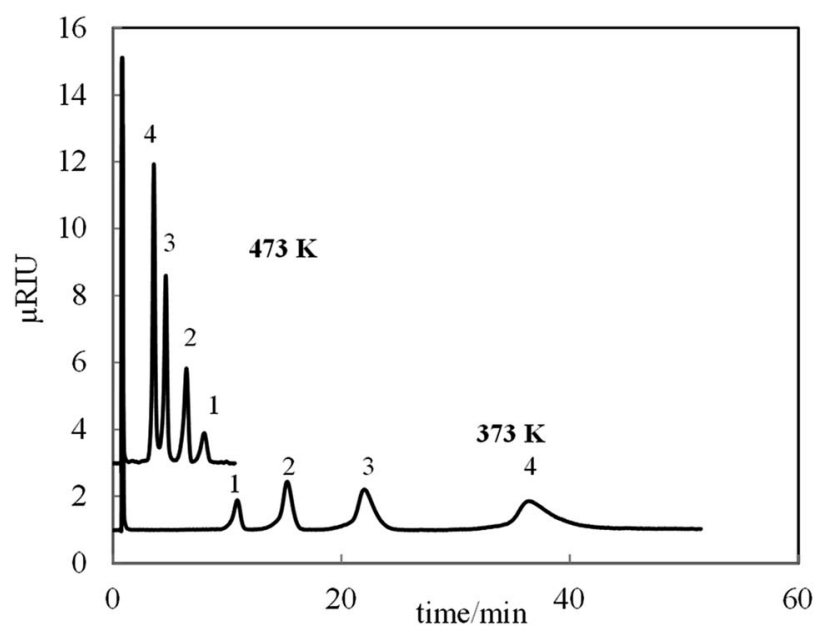

Fig. 4 Chromatograms of tetraalkylammonium ions on a $\mathrm{MCl}$ GEL CK10S column (4.6 mm I.D. $\times 50 \mathrm{~mm}$ ) obtained at $373 \mathrm{~K}$ and $473 \mathrm{~K}$. Eluent: $25 \mathrm{mM} \mathrm{Cs}_{2} \mathrm{SO}_{4}$. Detection: differential reflactometer. Flow

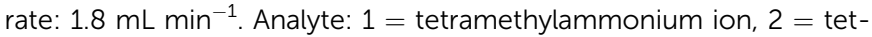
raethylammonium ion, $3=$ tetra- $n$-propylammonium ion, $4=$ tetra- $n$ butylammonium ion.

on the columns packed with MCI GEL CK02A and Nafion, respectively. These ion-exchange resins are not so stable that they decompose and lose the fixed ions over $403 \mathrm{~K}$ and $423 \mathrm{~K}$, respectively, probably due to relatively low cross-linking degree. Actually the other commercially available ion-exchange resins that we tried to use have even lower heat stability than MCI GEL CK02A and Nafion. We have thus carried out a series of experiments for MCI GEL CK02A and Nafion in the range of 313-390 $\mathrm{K}$. The upper temperature limits of the cation-exchange resins used in this study determined by measurements of the change in ion-exchange capacity are listed in Table 1 together with other characteristics of the resins. As shown in Fig. 5(b), the retention order of alkali metal ions on the Nafion column does not change in the temperature range examined. On the other hand, the reversal of the retention order for $\mathrm{Li}^{+}$and $\mathrm{Na}^{+}$was observed at $340 \mathrm{~K}$ for the MCI GEL CK02A column. However, the change in retention selectivity with temperature is much smaller than that observed on the MCI GEL CK10S column. These results indicate that the change in the hydration structure of an analyte ion caused by the counterion $\left(\mathrm{Cs}^{+}\right)$does not take place in the solution but in the ion-exchange resin.

It has been reported that the number of water molecules hydrated to some ions such as $\mathrm{Na}^{+}$and $\mathrm{H}_{2} \mathrm{PO}_{4}{ }^{-}$decreases with an increase in the ion-exchange capacity and the cross-linking degree and this experimental result can be ascribed to dehydration of the ion by a decrease in the void space for the ions in the ion-exchange resins. ${ }^{22-26}$ The difference in the distribution coefficients of alkali metal ions between Nafion, MCI GEL CK02A and MCI GEL CK10S may also be attributed to differences in the ion-exchange capacity and crosslinking degree of these resins (Table 1). Since $\mathrm{Cs}^{+}$has relatively large ionic size and its concentration is larger in MCI GEL CK 10S than in CK02A and Nafion, the remaining void space should be smaller than in the other two resins. The counterion effect on the hydration structure of the analyte ion is thus expected to be enhanced in MCI GEL CK10S resin at elevated temperatures.

From the results shown above, it is expected that counter cations larger than $\mathrm{Cs}^{+}$may cause more drastic change in retention of alkali metal ions. Fig. 6(a) and (b) demonstrate the $\ln K_{\mathrm{D}}$ vs. $1 / T$ plots for alkali metal ions obtained on MCI GEL CK02A and Nafion columns by elution with $50 \mathrm{mM}$ tetramethylammonium bromide (TMABr) aqueous solution. The counterion $\left(\mathrm{Na}^{+}\right)$of MCI GEL CK10S could not completely be replaced by $\mathrm{TMA}^{+}$probably because $\mathrm{TMA}^{+}$is partly excluded from the pores of the resin owing to the relatively large size of $\mathrm{TMA}^{+}$and high cross-linking degree or small pore size of the resin. As can be seen from the comparison of Fig. 6(a) with Fig. 5(a), the $K_{\mathrm{D}}$ value of $\mathrm{Li}^{+}$on MCI GEL CK02A is larger not only than the value for $\mathrm{Na}^{+}$but also than that for $\mathrm{K}^{+}$even at ambient temperature and becomes larger than the value for $\mathrm{Rb}^{+}$ at $320 \mathrm{~K}$ or above in the TMABr elution system. On the Nafion column, $\mathrm{Li}^{+}$also has the larger $K_{\mathrm{D}}$ value than $\mathrm{Na}^{+}$by elution with the TMABr aqueous solution although the difference in $K_{\mathrm{D}}$ value between $\mathrm{Li}^{+}$and $\mathrm{Na}^{+}$is rather small probably because of the low ion-exchange capacity (Fig. 6(b)). These results indicate that the relative size of the background counterion with respect to the inner pore size of the ion-exchange resin as well as the hydration energy governs the ion-exchange selectivity.

\section{Effects of temperature and counterion on the swelling of the cation-exchange resin}

The swelling or water absorbing properties of the cationexchange resin in different counterion forms were evaluated by measuring the volumes of water-swollen ion-exchange resin and water absorbed in the resin at different temperatures in order to further investigate the origin of the effect of counterion on the temperature dependence of the ion-exchange equilibria. The volume of the water-swollen ion-exchange resin $\boldsymbol{V}_{\text {IER }}$ was determined by a chromatographic method using a stainless 

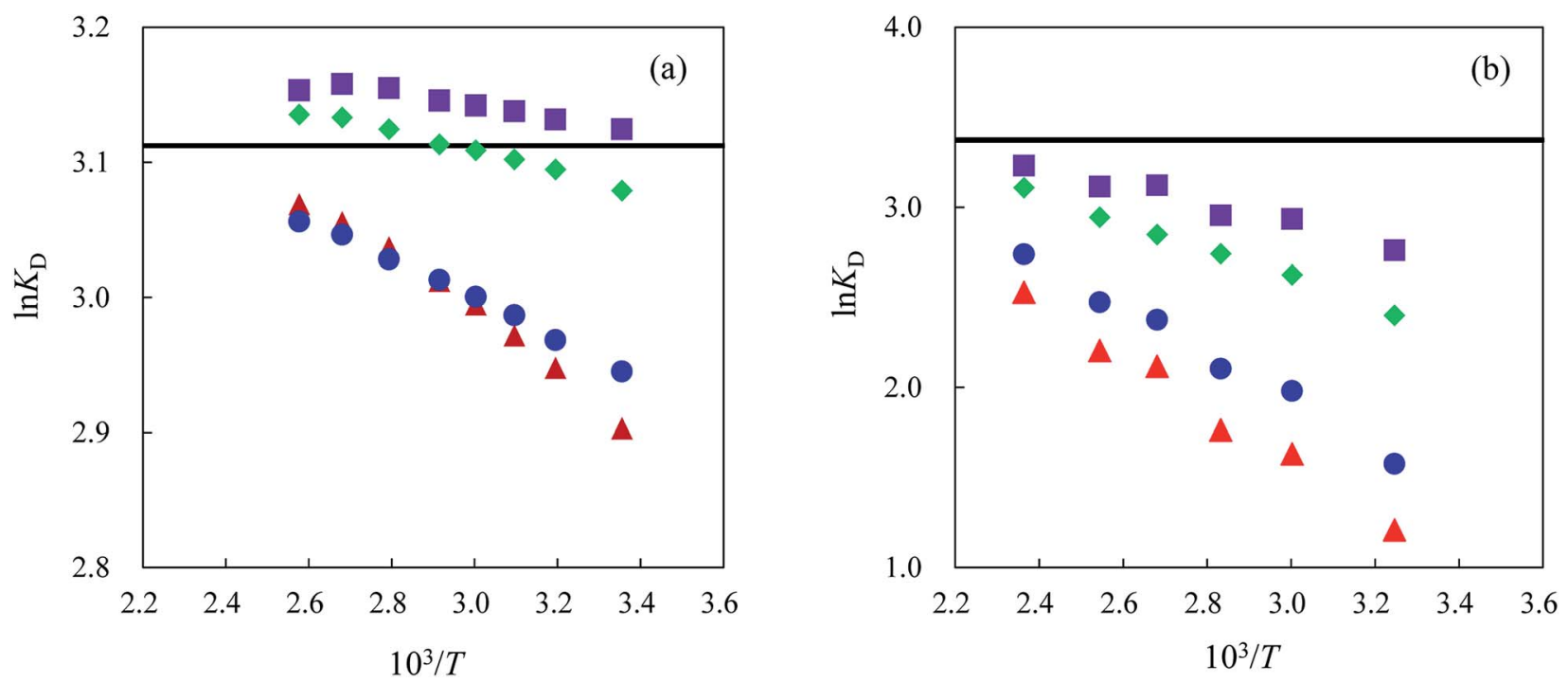

Fig. 5 Plots of In $K_{\mathrm{D}}$ for alkali metal ions as a function of reciprocal of temperature on a $\mathrm{MCl}$ GEL CKO2A column (a) and a Nafion column (b). Eluent: $25 \mathrm{mM} \mathrm{Cs}_{2} \mathrm{SO}_{4}$. Symbols: $(\boldsymbol{\Delta}) \mathrm{Li}^{+},(\bullet) \mathrm{Na}^{+},(\diamond) \mathrm{K}^{+},(\square) \mathrm{Rb}^{+}$. The solid line denotes the $\mathrm{ln} K_{\mathrm{D}}(\mathrm{E})$ value for each column.

steel column packed with the resin according to the following equation:

$$
V_{\mathrm{IER}}=\boldsymbol{V}_{\mathrm{t}}-\boldsymbol{V}_{\mathrm{int}}
$$

where $\boldsymbol{V}_{\text {int }}$ is the interstitial or interparticulate volume. The $\boldsymbol{V}_{\text {int }}$ values were obtained from measurements of the retention volume of Blue Dextran 2000, which one can regard as a compound that is completely excluded from the network of the resin by steric exclusion.

The change in $\boldsymbol{V}_{\text {IER }}$ with temperature in the range of $308 \mathrm{~K}$ to $433 \mathrm{~K}$ for alkali metal forms of MCI GEL CK10S is shown in Fig. 7. It is noteworthy that the $V_{\text {IER }}$ values for the $\mathrm{K}^{+}, \mathrm{Rb}^{+}$and $\mathrm{Cs}^{+}$forms of the resin gradually increase with an increase in temperature, while those for the resins in $\mathrm{Li}^{+}$and $\mathrm{Na}^{+}$forms decrease. Especially the resin in $\mathrm{Li}^{+}$form shows remarkable decrease of $\boldsymbol{V}_{\text {IER }}$ with an increase in temperature. On the contrary, the $\boldsymbol{V}_{\mathrm{s}}$ value for each alkali metal form of the resin was approximately constant independent of temperature as shown in Fig. 8. The $\boldsymbol{V}_{\text {IER }}$ can be given by the sum of the volumes of hydrated metal counterion $\boldsymbol{V}_{\mathrm{HM}}$ and fixed sulfonate ion $\boldsymbol{V}_{\mathrm{HF}}$, the resin polymer matrix $\boldsymbol{V}_{\mathrm{P}}$ and the free water in the resin $\boldsymbol{V}_{\mathrm{W}}$ as follows:

$$
V_{\mathrm{IER}}=\boldsymbol{V}_{\mathrm{HM}}+\boldsymbol{V}_{\mathrm{HF}}+\boldsymbol{V}_{\mathrm{P}}+\boldsymbol{V}_{\mathrm{W}}
$$

on the other hand, $\boldsymbol{V}_{\mathrm{S}}$ is represented by the sum of the volumes of the polymer matrix and the nonhydrated metal ion $\boldsymbol{V}_{\mathrm{M}}$ and sulfonate ion $\boldsymbol{V}_{\mathrm{F}}$ as:

$$
V_{\mathrm{s}}=V_{\mathrm{M}}+\boldsymbol{V}_{\mathrm{F}}+\boldsymbol{V}_{\mathrm{P}}
$$

it can thus be concluded that $\boldsymbol{V}_{\mathrm{P}}$ as well as $\boldsymbol{V}_{\mathrm{M}}$ and $\boldsymbol{V}_{\mathrm{F}}$ are constant regardless of the change in temperature and the difference in temperature dependence of $\boldsymbol{V}_{\text {IER }}$ between the counterion forms of the resin is attributed to the change in the $\boldsymbol{V}_{\mathrm{HM}}$ and $\boldsymbol{V}_{\mathrm{W}}$ with temperature.

We have shown that the hydration number of $\mathrm{Rb}^{+}$as well as $\mathrm{Sr}^{2+}$ present as the counterions of fixed sulfonate groups in MCI GEL CK10S slightly decreases with an increase in temperature similarly to the changes observed for their aqueous solutions. ${ }^{19}$ The thermal motion of the resin polymer chains would be intensified at higher temperatures, leading to expansion of the resin network or increase in $\boldsymbol{V}_{\mathrm{W}}$. Therefore, the decrease in $\boldsymbol{V}_{\text {IER }}$ of the cation-exchange resin in $\mathrm{Li}^{+}$and $\mathrm{Na}^{+}$forms with an increase in temperature indicates that the hydration numbers of these metal ions in the resin remarkably decrease as the temperature rises compared to the other alkali metal ions.

The $V_{\text {IER }}$ of the resin in $\mathrm{Li}^{+}$form is the largest up to $403 \mathrm{~K}$, while that for the $\mathrm{Cs}^{+}$form resin becomes larger over $418 \mathrm{~K}$, which reveals that the swelling energy of the resin in $\mathrm{Cs}^{+}$form is

Table 1 Properties of the cation-exchange resins used in this study

\begin{tabular}{lllll}
\hline Resin & $\begin{array}{l}\text { Functional } \\
\text { group }\end{array}$ & $\begin{array}{l}\text { Cation-exchange } \\
\text { capacity }\left(\mathrm{meq} . \mathrm{mL}^{-1}\right)\end{array}$ & $\begin{array}{l}\text { Upper temperature } \\
\text { limit }{ }^{a}(\mathrm{~K})\end{array}$ & $\begin{array}{l}\text { Cross-linking } \\
\text { degree }^{b}(\%)\end{array}$ \\
\hline MCI GEL CK10S & $-\mathrm{SO}_{3}{ }^{-}$ & 2.2 & 473 & $\begin{array}{l}\text { Particle } \\
\text { diameter }^{b}\end{array}$ \\
MCI GEL CK02A & $-\mathrm{SO}_{3}{ }^{-}$ & 0.66 & 403 & 10 \\
Nafion & $-\mathrm{SO}_{3}{ }^{-}$ & 0.35 & 423 & 2 \\
\hline
\end{tabular}

${ }_{b}^{a}$ The highest temperature at which the decrease in cation-exchange capacity of the resin by elution with $25 \mathrm{mM} \mathrm{Na}_{2} \mathrm{SO}_{4}$ for $30 \mathrm{~h}$ is less than $5 \%$.

${ }^{b}$ Manufactures' data. 

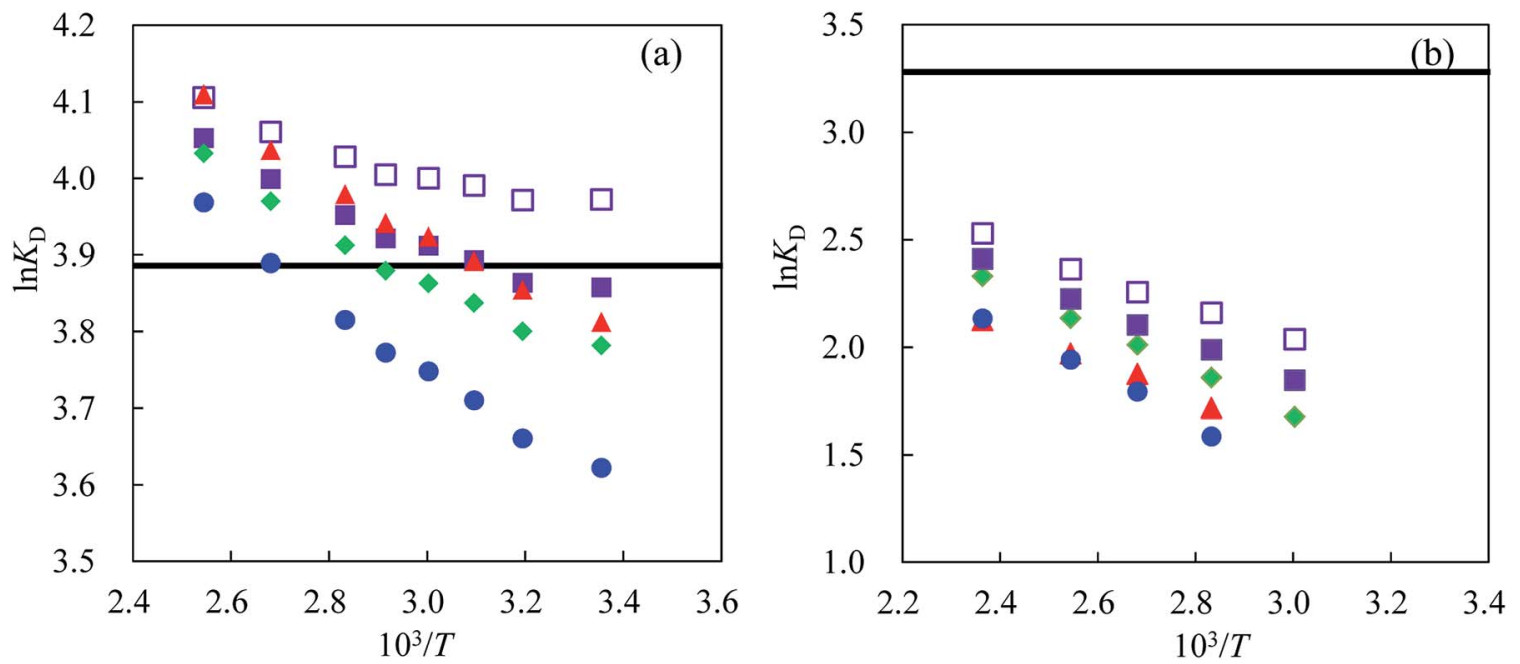

Fig. 6 Plots of In $K_{\mathrm{D}}$ for alkali metal ions as a function of reciprocal of temperature on a $\mathrm{MCl}$ GEL CKO2A column (a) and a Nafion column (b). Eluent: 50 mM TMABr. Symbols: $(\Delta) \mathrm{Li}^{+},(\bullet) \mathrm{Na}^{+},(\Delta) \mathrm{K}^{+},(\square) \mathrm{Rb}^{+},(\square) \mathrm{Cs}^{+}$. The solid line denotes the In $K_{\mathrm{D}}(\mathrm{E})$ value for each column.

larger than those for the other alkali metal forms of the cationexchange resin at $418 \mathrm{~K}$ or above. The swelling pressure $\Pi$ may be one of the important determinants of the ion-exchange selectivity as indicated by the Gregor equation: $:^{27-29}$

$$
\ln K_{\mathbf{A} / \mathbf{E}}=\frac{\Pi}{R T}\left(z_{\mathbf{A}} v_{\mathbf{E}}-z_{\mathbf{E}} v_{\mathbf{A}}\right)
$$

where $R$ is the gas constant and $v$ is the partial molar volume of the hydrated ionic species. This equation predicts that the ionexchanger prefers the ion with smaller solvated volume. This prediction is partly in accord with the results shown in Fig. 1 and 7 since $\mathrm{Li}^{+}$has the smallest distribution coefficient among the other alkali metal ions at lower temperatures, while at higher temperatures it preferentially distributes into the cation-exchange resin.

However, this simple model cannot completely account for the observed selectivity reversals with change in temperature because the temperature at which the reversal of the retention

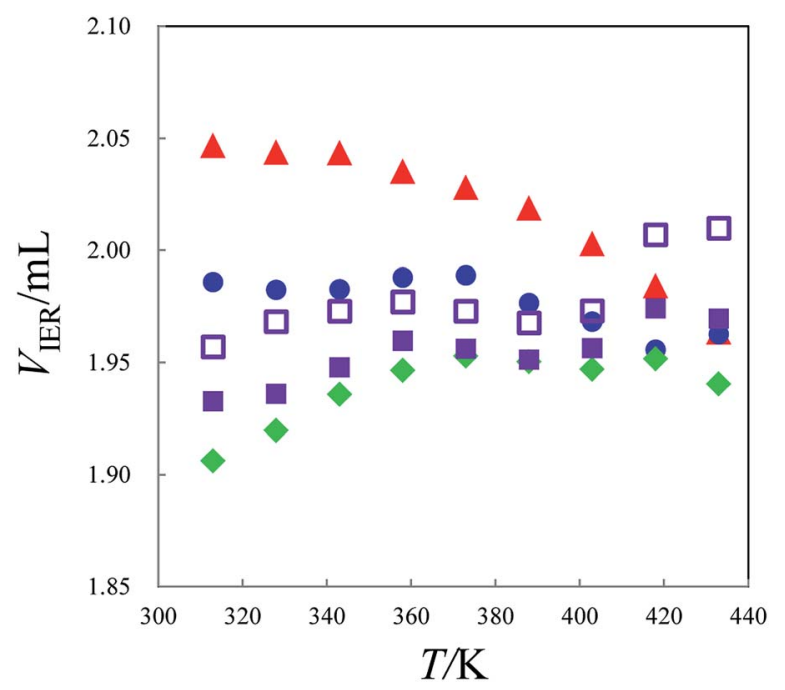

Fig. 7 Dependence of the $V_{\text {IER }}$ values on temperature for $\mathrm{MCl} G E L$ CK10S resin in alkali metal ion forms. Symbols: $(\Lambda) \mathrm{Li}^{+},(\bullet) \mathrm{Na}^{+},(\diamond) \mathrm{K}^{+}$, (घ) $\mathrm{Rb}^{+},(\square) \mathrm{Cs}^{+}$. order of alkali metal ions occurs depends on the background counterion in the resin. The chemical components of the Gregor's model are the hydrated ions as well as the resin matrix with its solvated fixed ions and the solvent. The hydration of the ions in the resin phase is assumed to be the same as that in the solution phase in this model. The results shown in Fig. 1 and 57 , however, reveal that the hydration structure of an analyte ion in the resin is different from that in the aqueous solution and it also depends on the nature of the background counterion of the ion-exchange resin. At elevated temperatures, the hydration energy of ions becomes smaller so that the cations with smaller ionic radii are easily dehydrated and selectively distribute into the cation-exchange resin. Large counterions present in high density in the resin may enhance the dehydration of the analyte ions at elevated temperatures, leading to quite different ionexchange selectivity from that observed at ambient temperature.

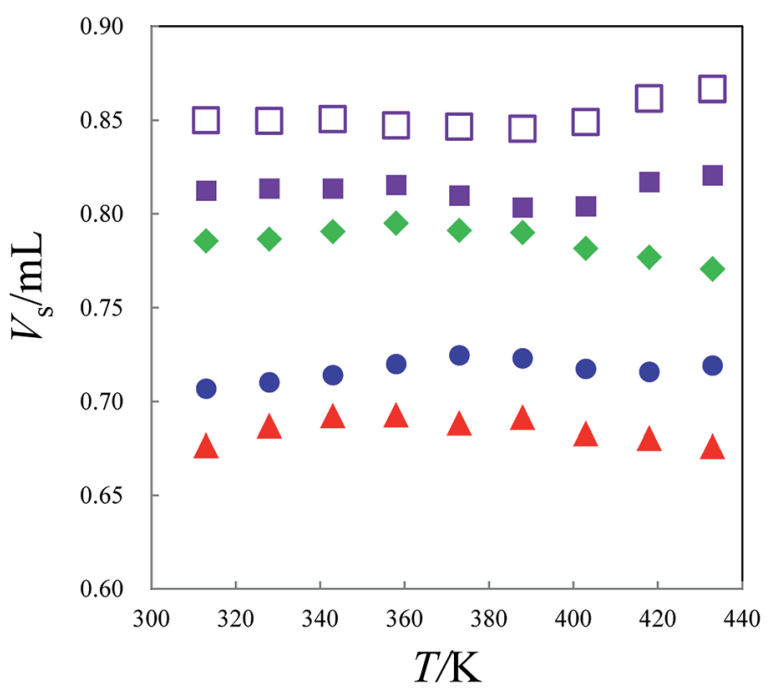

Fig. 8 Dependence of the $V_{\mathrm{s}}$ values on temperature for $\mathrm{MCl} \mathrm{GEL}$ CK10S resin in alkali metal ion forms. For other details see Fig. 7. 


\section{Conclusions}

We have made a new finding by SW-IEC that even relatively strongly hydrated small metal ions show a drastic change in ionexchange selectivity on a usual cation-exchange resin by synergistic effect of temperature and the nature of the background counterion in the aqueous solution. With the ion-exchange resin of higher ion-exchange capacity and cross-linking degree, large sized counterions such as $\mathrm{Cs}^{+}$and tetramethylammonium ion cause a drastic change in retention order from $\mathrm{Li}^{+}<\mathrm{Na}^{+}<\mathrm{K}^{+}<\mathrm{Rb}^{+}$in water at ordinary temperature to $\mathrm{Rb}^{+}<\mathrm{K}^{+}$ $<\mathrm{Na}^{+}<\mathrm{Li}^{+}$in superheated water. These observations can be ascribed to the change in local hydration structures of the ions in the resin phase due to dehydration enhanced by interionic contacts with a large coexisting counterion and lower ionic hydration energy at elevated temperatures.

These results suggest that even at ambient temperature the separation of analyte ions in ion-exchange processes is affected by the nature of the coexisting counterions. It has been well known that larger separation factor is usually obtained with weaker counterions. ${ }^{7}$ For example, the difference in the distribution coefficient between analyte cations is better with $\mathrm{Li}^{+}$ counterion than with $\mathrm{Cs}^{+}$at ambient temperature. The mechanism presented in this study may explain this phenomenon, which has not been successfully interpreted yet. In other words, the change in the hydration of the exchanging ions upon their transition from the solution phase to the resin phase or vice versa may play a predominant role in determining the ionexchange selectivity. SW-IEC with the aqueous mobile phase containing large eluting ions may also bring about drastic change in separation selectivity and can improve the separation of ionic solutes through temperature programming.

\section{Conflicts of interest}

There are no conflicts to declare.

\section{Acknowledgements}

This research was supported by a Grant-in-Aid for Scientific Research No. 20350034 from Ministry of Education, Culture, Sports, Science and Technology, Japan.

\section{References}

1 R. W. Grimshaw and C. E. Harland, Ion-Exchange: Introduction to Theory and Practice, The Chemical Society, London, 1975.
2 P. G. Rigas, Instrum. Sci. Technol., 2012, 40, 161-193.

3 F. Helfferich, Ion Exchange, McGraw-Hill, New York, 1962.

4 J. S. Fritz, J. Chromatogr. A, 2005, 1085, 8-17.

5 G. Stahlberg, Anal. Chem., 1994, 66, 440-449.

6 T. Okada, Anal. Sci., 1998, 14, 469-477.

7 T. Okada, Anal. Chem., 1998, 70, 1692-1700.

8 T. Okada, J. Chromatogr. A, 1999, 850, 3-8.

9 S. Afrashtehfar and F. F. Cantwell, Anal. Chem., 1982, 54, 2422-2427.

10 R. A. Hux and F. F. Cantwell, Anal. Chem., 1984, 56, 12581263.

11 H. Liu and F. F. Cantwell, Anal. Chem., 1991, 63, 993-1000.

12 H. Liu and F. F. Cantwell, Anal. Chem., 1991, 63, 2032-2037.

13 A. I. Liapis and B. A. Grimes, J. Sep. Sci., 2005, 28, 1909-1926.

14 M. Shibukawa, T. Shimasaki, S. Saito and T. Yarita, Anal. Chem., 2009, 81, 8025-8032.

15 M. Shibukawa, A. Taguchi, Y. Suzuki, K. Saitoh, T. Hiaki and T. Yarita, Analyst, 2012, 137, 3154-3159.

16 M. Shibukawa, R. Morinaga and S. Saito, Bunseki Kagaku, 2016, 65, 615-623.

17 N. E. Fortier and J. S. Fritz, Talanta, 1987, 34, 415-418.

18 P. Hatsis and C. A. Lucy, Analyst, 2001, 126, 2113-2118.

19 M. Shibukawa, M. Harada, T. Okada, Y. Ogiyama, T. Shimasaki, Y. Kondo, A. Inoue and S. Saito, RSC Adv., 2012, 2, 8985-8991.

20 E. R. Nightingale, J. Phys. Chem., 1959, 63, 1381-1387.

21 R. L. David, CRC Handbook of Chemistry and Physics, CRC Press, Boca Raton, 84th edn, 2004.

22 A. Yuchi, S. Kuroda, M. Takagi, Y. Watanabe and S. Nakao, Anal. Chem., 2010, 82, 8611-8617.

23 Y. Watanabe, K. Ohnaka, S. Fujita, M. Kishi and A. Yuchi, Anal. Chem., 2011, 83, 7480-7485.

24 D. Nandan, B. Venkataramani and A. R. Gupta, Langmuir, 1993, 9, 1786-1793.

25 R. W. Creekmore and C. N. Reilley, Anal. Chem., 1970, 42, 570-575.

26 H. D. Sharma and N. Subramanian, Can. J. Chem., 1971, 49, 3948-3955.

27 H. P. Gregor, J. Am. Chem. Soc., 1948, 70, 1293.

28 H. P. Gregor, J. Am. Chem. Soc., 1951, 73, 642-650.

29 H. P. Gregor and J. I. Bregman, J. Colloid Sci., 1951, 6, 323347.

30 http://www.spiraxsarco.com/esc/SW_Properties.aspx. 